\title{
Aplasia congénita unilateral del cartílago alar
}

\section{Unilateral congenital aplasia of the alar cartilage}

\author{
Jaime Osorio M. ${ }^{1,2}$, Alfredo Urrutia F. ${ }^{1}$, Angélica Suazo D. ${ }^{3}$, Daniel Cantero C. ${ }^{1,2}$
}

\begin{abstract}
Resumen
El objetivo de este trabajo es discutir la presentación y el manejo de la aplasia congénita unilateral del cartílago alar. En este informe, presentamos el caso de un paciente sometido a rinoseptoplastia abierta primaria que presenta aplasia congénita del cartílago alar izquierdo que se repara con cartílago septal. Se discute el manejo de casos y se revisa la literatura. Las anomalías nasales congénitas son poco frecuentes. La ausencia aislada de cualquier estructura nasal específica es aún más rara. El diagnóstico preoperatorio en una nariz hispana es difícil, pero cuando se encuentra este defecto congénito, puede repararse con cartílago septal u otros tipos de cartílago, dependiendo de la disponibilidad del injerto, con buenos resultados. Se recomienda un enfoque abierto para este tipo de patología. La aplasia unilateral del cartílago alar podría reconstruirse con éxito utilizando el cartílago septal a través de un abordaje abierto.
\end{abstract}

Palabras clave: Cartílago alar, aplasia, trastornos nasales congénitos, cirugía plástica facial y reconstructiva, rinoseptoplastia.

\begin{abstract}
The objective of this work is discussing the presentation and management of unilateral congenital aplasia of the alar cartilage. In this report, we present the case of a patient undergoing primary open rhinoseptoplasty presenting congenital aplasia of the left alar cartilage that is repaired with septal cartilage. Case management is discussed, and the literature is reviewed. Congenital nasal abnormalities are rare. The isolated absence of any specific nasal structure is even more rare. Preoperative diagnosis in a Hispanic nose is difficult, but when this deformed congenital is found, it can be repaired with septal cartilage or other types of cartilage, depending on the availability of the graft, with good results. An open approach is recommended for this type of pathology. Unilateral alar cartilage aplasia could be successfully reconstructed using septal cartilage through and open approach.

Keywords: Alar cartilage, aplasia, congenital nasal disorders, facial plastic and reconstructive surgery, rhinoseptoplasty.
\end{abstract}

'Servicio de Otorrinolaringología Hospital Barros Luco Trudeau. Santiago, Chile. ${ }^{2}$ Departamento de Otorrinolaringología de la Universidad de Chile. Santiago, Chile.

${ }^{3}$ Escuela de Medicina. Universidad Católica de la Santísima Concepción. Concepción, Chile.

Los autores declaran no tener conflictos de interés.

Recibido el 5 de julio de 2020 Aceptado el 20 de octubre de 2020.

Correspondencia: Jaime Osorio M. Gran Avenida José Miguel Carrera 3204, San Miguel Santiago, Chile. Email: jaime_osorio@yahoo. com

\section{Introducción}

Las anomalías congénitas de la nariz son raras y ocurren en $1 / 20.000$ a 1/40.000 recién nacidos ${ }^{1}$. Se cree que los defectos faciales surgen debido a deficiencias en el desarrollo de las células de la cresta neural, que pueden incluir migración mesenquimatosa insuficiente, necrosis celular o disminución de la proliferación celular, y pueden ser causadas por factores genéticos o ambientales ${ }^{2}$.

En una revisión de 261 pacientes, Losee y cols. ${ }^{1}$ clasificaron las anomalías nasales en las siguientes cuatro categorías:
- hipoplasia y atrofia.

- hiperplasia y duplicaciones.

- hendiduras.

- neoplasias y anomalías vasculares.

El cartílago alar o cartílago lateral inferior es una estructura doble que soporta la punta nasal, evita el colapso de las válvulas nasales externas y juega un papel importante en la determinación del contorno cosmético de la nariz y las fosas nasales ${ }^{3}$. La literatura contiene solo unos pocos casos documentados de ausencia congénita no sindrómica de un solo componente de cartílago de la nariz ${ }^{4}$. Describi- 
mos un caso de aplasia congénita del cartílago alar izquierdo en un paciente que se sometió a rinoseptoplastia primaria abierta.

\section{Caso Clínico}

Paciente femenina de 46 años sin antecedentes médicos relevantes, derivada por obstrucción nasal, colapso de la válvula nasal izquierda, giba prominente, punta nasal bulbosa ligeramente desviada hacia la derecha, desviación septal izquierda y asimetría de las fosas nasales. No se informaron traumatismos nasales ni procedimientos quirúrgicos previos. El examen físico inicial mostró piel gruesa pero no evidencia clara de deformidad congénita (Figura 1).
Se realizó rinoseptoplastia abierta primaria. Intraoperatoriamente, se observó aplasia del cartílago alar izquierdo sin la presencia de ninguna de sus tres porciones (cura medial, intermedio y lateral) (Figura 2).

El cartílago septal se obtiene para utilizarse como injerto, dejando un marco septal de un centímetro. El cartílago alar izquierdo ausente se reconstruyó utilizando cartílago septal. Se usó una tira de cartílago de 5 x $25 \mathrm{~mm}$ para reconstruir el cartílago alar. Se realizaron incisiones de cartílago parcial debilitante para agregar flexibilidad al cartílago. Neo-domo se realizó con sutura intradomal e interdomal con prolene 5/0. Se coloca una tira de injerto de cartílago septal debajo de las cruras laterales (alar strut grafts) y se asegura con prolene 5/0 (Figura 2). La cirugía se completa con la

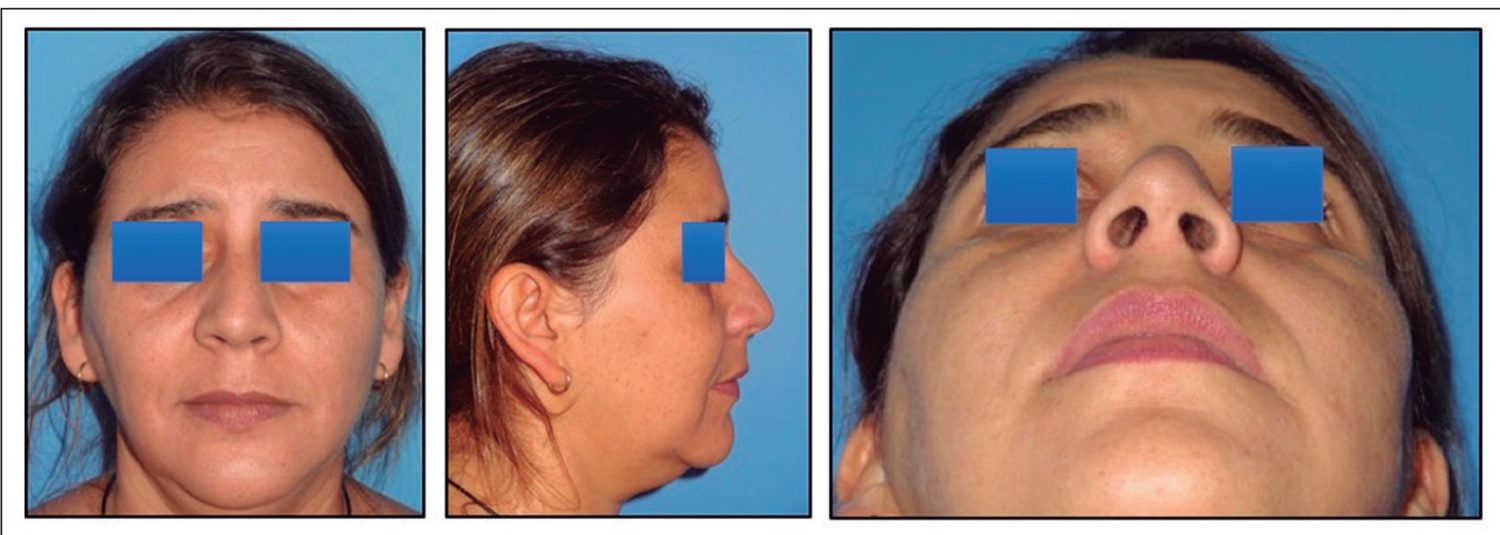

Figura 1. A) Proyección frontal. B) Proyección lateral. C) Visión inferior de pirámide nasal donde se observa leve asimetría de fosas nasales.

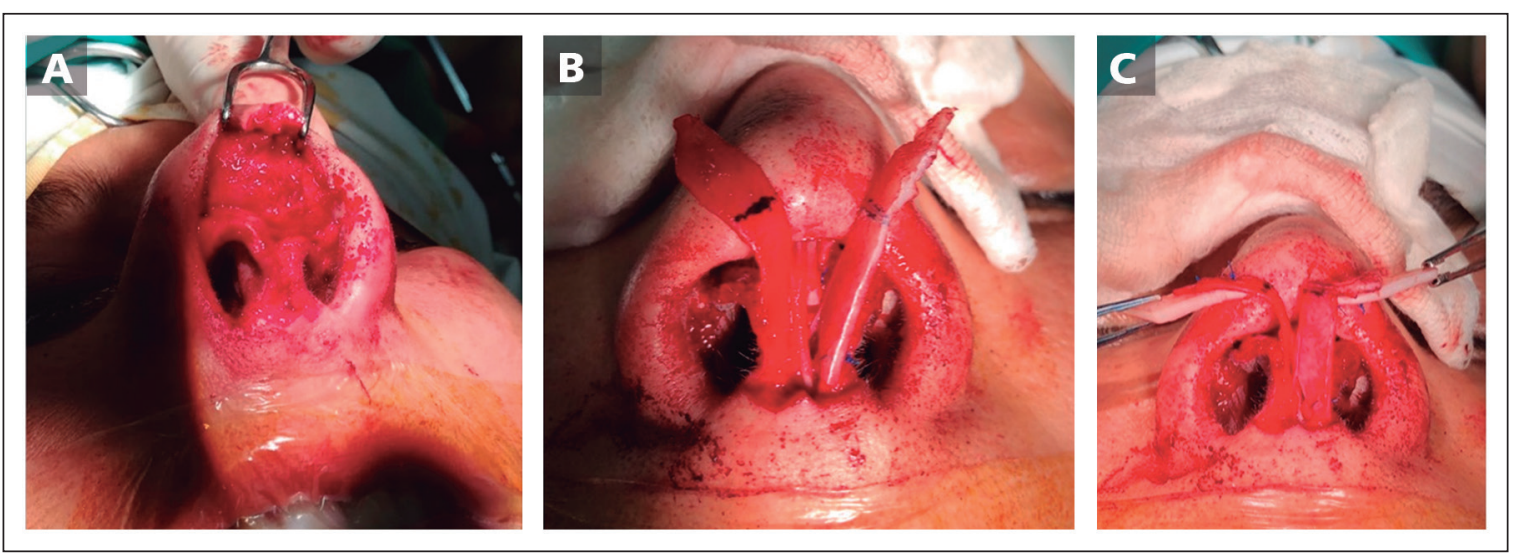

Figura 2. A) Se observa aplasia de cartílago alar medial, intermedio y lateral izquierdo. B) Dando forma al injerto de cartílago septal. C) Posición final de cartílago septal reparando aplasia de cartílago alar izquierdo. 


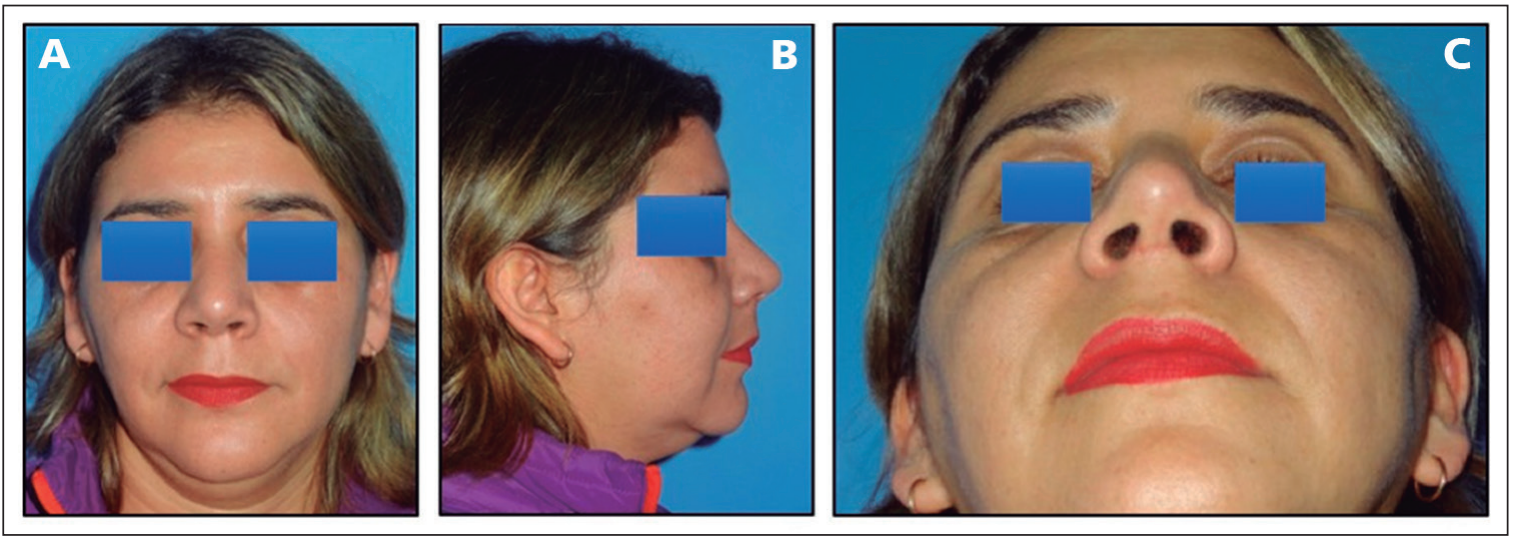

Figura 3. Fotos tomadas a los 6 meses posterior a la cirugía. A) Proyección frontal. B) Proyección lateral donde se observa ausencia de giba osteocartilaginosa. C) Proyección inferior donde se observa simetría de ambas narinas.

resección de la giba osteocartilaginosa, las osteotomías media y lateral y la colocación de injertos de extensión. El injerto de cartílago septal triturado se coloca para camuflar las irregularidades de la punta nasal.

Después de la cirugía, se obtuvo un buen resultado funcional y estético. La asimetría de la punta se corrigió con una proyección y rotación adecuadas. La obstrucción nasal mejoró y la paciente quedó satisfecha con los resultados de la operación como muestran las fotos registradas a los 6 meses posoperatorias (Figura 3).

\section{Discusión}

Hasta donde sabemos, solo tres casos de aplasia congénita del cartílago alar han sido publicados en la literatura mundial. Esta malformación es extremadamente infrecuente. Sin embargo, es un hallazgo para tener en cuenta antes de realizar una rinoplastia, especialmente porque el diagnóstico preoperatorio puede ser difícil. En nuestro caso particular, aunque la malformación no fue evidente a primera vista, el colapso unilateral de la válvula nasal fue un hallazgo que podría haber sugerido la presencia de esta anomalía. De hecho, este escenario representa un desafío para el cirujano para reconstruir un contorno armónico de la punta nasal manteniendo o mejorando la funcionalidad por lo cual es mejor conocerlo antes de la cirugía $a^{5-8}$.
La nariz latinoamericana o hispana que predomina en Chile se caracteriza por tener un soporte de punta débil, estructuras cartilaginosas de punta delgada, huesos nasales cortos y piel gruesa ${ }^{9}$. Todas estas características dificultan el análisis de los cartílagos alar en el período preoperatorio. El examen físico sigue siendo la mejor herramienta para evaluar la punta nasal del paciente candidato a rinoplastia mediante la inspección y palpación de los cartílagos nasales. Aunque la tomografía computarizada (TC) es una herramienta muy útil para evaluar el tabique nasal y las cavidades paranasales, no es muy precisa al evaluar los cartílagos de las puntas. En el caso de nuestro paciente, la desviación septal y la piel gruesa dificultaron el análisis clínico de los cartílagos alar preoperatoriamente $e^{5-8}$. Una vez que se realizó el diagnóstico intraoperatorio, tuvimos que decidir el tipo de injerto para la reconstrucción. Para reconstruir el cartílago alar, se requiere una tira de cartílago de al menos $5 \mathrm{~mm}$ de ancho y $25 \mathrm{~mm}$ de largo, que debe modelarse y suturarse con proleno 5/0 para darle el contorno nasal adecuado. Dado que este caso era una rinoseptoplastia primaria, había mucho cartílago septal disponible. El cartílago septal tiene la rigidez adecuada y se puede recortar y modelar para reemplazar el cartílago alar ${ }^{7}$. Sin embargo, el cartílago septal no siempre está disponible. El cartílago de la oreja es una buena alternativa debido a la curvatura natural de la concha ${ }^{10}$, pero también se utiliza cartílago costal. Sin embargo, la extracción del cartílago de la oreja o costilla aumenta el tiempo 
quirúrgico y las posibilidades de complicaciones quirúrgicas ${ }^{5-8}$.

Realizamos un abordaje abierto de rinoplastia para manejar mejor la punta nasal. La visualización directa de las estructuras de las puntas facilitó el diagnóstico y el tratamiento de este caso particular. Creíamos que un enfoque cerrado sería factible, pero sería técnicamente más difícil reconstruir el cartílago alar completo a través de un enfoque cerrado.

Además, a nuestro paciente se le colocó un injerto de cartílago septal debajo de la crura lateral para brindar más soporte a la válvula nasal interna ${ }^{5-8}$. El resultado exitoso en esta rara condición se logró a través de la rinoseptoplastia de abordaje abierto combinada con injerto de cartílago septal.

\section{Conclusión}

Aunque es poco frecuente, los cirujanos que realizan rinoseptoplastias deben ser conscientes de las deformidades congénitas de la punta nasal. La aplasia unilateral del cartílago alar podría reconstruirse con éxito utilizando el cartílago septal a través de un abordaje abierto.

\section{Bibliografía}

1. Losee JE, Kirschner RE, Whitaker LA, et al. Congenital nasal anomalies: a classification scheme. Plast Reconstr Surg. 2004;113:676-689.
2. Millard DR Jr. Embryonic rationale for the primary correction of classical congenital clefts of the lip and palate. Ann R Coll Surg Engl. 1994;76(3):150160.

3. Janis JE, Trussler A, Ghavami A, Marin V, Rohrich RJ, Gunter JP. Lower lateral crural turnover flap in open rhinoplasty. Plast Reconstr Surg. 2009; 123:18301841.

4. Barutca SA, Öreroğlu AR, Usçetin I, et al. Isolated congenital partial absence of the left lower lateral nasal cartilage: case report. Ann Plast Surg. 2011;67: 662-664.

5. Kosins AM, Daniel RK, Sajjadian A, Helms J. Rhinoplasty: congenital deficiencies of the alar cartilage. Aesthet Surg J. 2013;33(6):799-808. doi: $10.1177 / 1090820 X 13495692$.

6. Temiz G, Yeşiloğlu N, Sarici M, Filinte GT. Congenital isolated aplasia of lower lateral cartilage and reconstruction using dorsal hump material. $J$ Craniofac Surg. 2014;25(5):e411-e413. doi: 10.1097/ SCS.0000000000000909.

7. Adelson RT, Karimi K, Herrero N. Isolated congenital absence of the left lower lateral cartilage. Otolaryngol Head Neck Surg. 2008;138(6):793-794. doi: 10.1016/j. otohns.2008.02.018.

8. Constantian MB. Elaboration of an alternative, segmental, cartilage-sparing tip graft technique: experience in 405 cases. Plast Reconstr Surg. 1999;103(1):237-254. doi: 10.1097/00006534199901000-00038.

9. Cobo R. Hispano/Rinoplastia Mestiza. Facial Plast Surg Clin N Am. 2010;18:173-188.

10. Bran G, Riedel F. Dome Reconstruction in a Rare Case of Alar Cartilage Agenesis of the Middle and Lateral Crura. Aesth Plast Surg. 2016; 40(5):685-9. 